\title{
Auf ein Bier mit Kommissar Maigret
}

\section{Julika Griem}

Krise und Gewohnheit scheinen sich auf einen ersten Blick als singuläres Ereignis und wiederholtes Tun gegenüberzustehen. Aber die Ausrufung einer Krise - wie z. B. jener der Geisteswissenschaften - kann auch zur Gewohnheit werden. Und manche Krisen - wie z. B. jene des Klimas - sind zwar längst als lebensbedrohlich anerkannt, doch trotzdem gelingt es uns nicht, schädliche Gewohnheiten zu ändern. Was könnten wir aus der gegenwärtigen Lage über die Bedeutung von Gewohnheiten lernen? Stabilisieren sie soziales Leben oder hemmen sie Reformfähigkeit? Wiegen sie uns in denkfauler Sicherheit oder schaffen sie die Entlastung, die Kontingenzbewusstsein erst hervorbringt? Wie wäre zwischen sinnvollen und sinnwidrigen Routinen zu unterscheiden? Und lassen sich neue Gewohnheiten über Nacht entwickeln, oder brauchen sie Zeit, um sich einzunisten, in Körper und Gruppen, Alltage und Lebensräume? Wie viel Zeit - wenn die Zeit, krisenbedingt, knapp bemessen ist?

Mit der Pandemie veränderte sich auch in Deutschland über Nacht vieles, was man eben gerade so machte - nicht nur Arbeiten, Wirtschaften, Regieren, Forschen und Lehren, sondern auch Feiern, Spielen und Ausruhen, Rausgehen und Drinbleiben. "Ways of doing things“ im Großen und Kleinen, über die wir nicht nachdenken, weil sie zu funktionieren scheinen: Parteitage und Pressekonferenzen, der Radiosender am Morgen, Lektüre oder anderes zum Einschlafen. Auch beim Lesen interessieren sich viele von uns nicht nur für singuläre Herausforderungen, sondern für die Wiederkehr des Bekannten - wir kennen die Spielregeln des Genres und genießen die minimale Variation, oder auch nur das vorhersehbar Eintretende. Man könnte nun glauben, dass in der Krise, angesichts der Suspendierung so vieler gut eingespielter Routinen, bewährte Lektüre-Gewohnheiten Sicherheit und Trost spenden.

Man könnte aber auch eine neue Gewohnheit erfinden. Ich habe im März, mit beginnender Einschränkung unserer öffentlichen und privaten Lebensvollzüge, intuitiv und plötzlich beschlossen, Georges Simenons 75 Maigret-Romane zu lesen. Ich interessiere mich zwar schon lange für Kriminalliteratur, hatte diesen Brocken aber bisher ausgespart. Die Entscheidung für Simenon fiel nicht als Einlösung eines lang gehegten Bildungsvorhabens: Endlich den gesamten Thackeray, alles von Stifter oder noch mal Proust und die Josephs-Romane; also jene Lektüre-Projekte im zwangsentschleunigten Modus, von denen Buchhändler*innen mit einer bildungsbürgerlich geprägten Stammkundschaft jetzt berichten. In dieses Schema passt der belgisch/französische Krimi-Klassiker nicht. Simenon wurde zwar von André Gide und Federico Fellini als literarischer Gigant empfohlen, schrieb aber doch 
in vieler Hinsicht schnell zu konsumierende Unterhaltungsliteratur, die ihre Leser*innen nicht zwingt, sich mit dem ästhetischen Eigensinn von Sprache zu befassen.

Die Maigret-Reihe (publiziert zwischen 1931 und 1972) verschafft mir inmitten der Pandemie keine professionell gestützte Versenkung, sondern eine unterhaltsam-beruhigende Verbindung von „Arbeit und Struktur“.In einer Zeit, in der sich eingespielte Zeitgefühle und Zeit-Regime aufzulösen beginnen, liefert das MaigretProjekt eine neue kleine Ordnung. Da ich über mehr Lesezeit als viele andere verfüge, kann ich versuchen, alle zwei Tage einen Roman zu beenden. Dieser Rhythmus korrespondiert mit der Struktur und Entstehung der Maigret-Krimis: Ihre Länge bemisst sich in allen 75 Fällen auf 175-195 Seiten (die 28 Maigret-Erzählungen sind

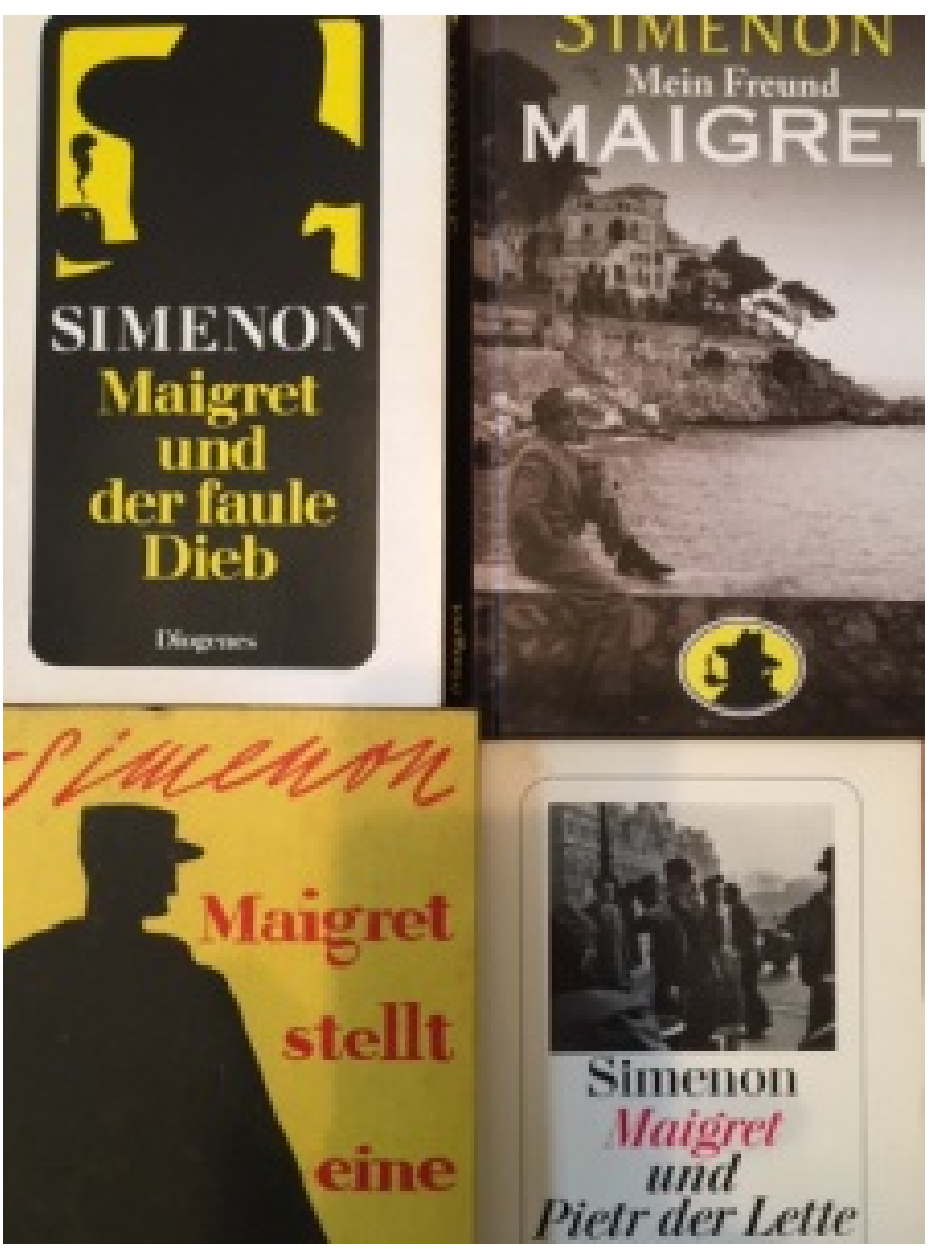
kürzer), und Simenon, als Journalist und Verfasser von pulp fiction geschult, hat sie in nahezu industrialisierter Serien-Produktion hergestellt - eine Woche pro Roman, und nicht mehr als drei Tage für die Überarbeitung. Um die gesuchte Gleichförmigkeit meiner Lektüre zu konterkarieren, habe ich ein Element der Kontingenz in den Selbstversuch eingebaut: Ich lese die Maigret-Romane nicht in der Reihenfolge ihres Erscheinens, was am einfachsten durch den Erwerb einer Gesamtausgabe einzulösen wäre. Meine ,Maigrets' kommen als gebrauchte Exemplare per Post, zusammengesucht über Online-Anbieter. So ein ,Tauschhandel' nicht nur von Büchern findet derzeit auch vermehrt in Stadtteilschränken und Hauseingängen statt. Er ermöglicht Einblicke in Lesegewohnheiten: Ich entledige mich der philologischen Routine, ein ,Werk ${ }^{6}$ chronologisch zu erschließen, lerne etwas über meinen Sammeltrieb und finde Spuren einer Gebrauchsgeschichte von Simenon-Leser*innen, die bei meinen ZufallsKäufen in Form von Geruch und Vergilbungsgrad, Anmerkungen und Aufklebern, Umschlaggestaltungen und Übersetzungen erkennbar werden. Offen ist noch, wie lange ich brauchen werde, um alle 75 Romane beschafft und gelesen zu haben - und ob mein Versuch überhaupt abgeschlossen wird. 


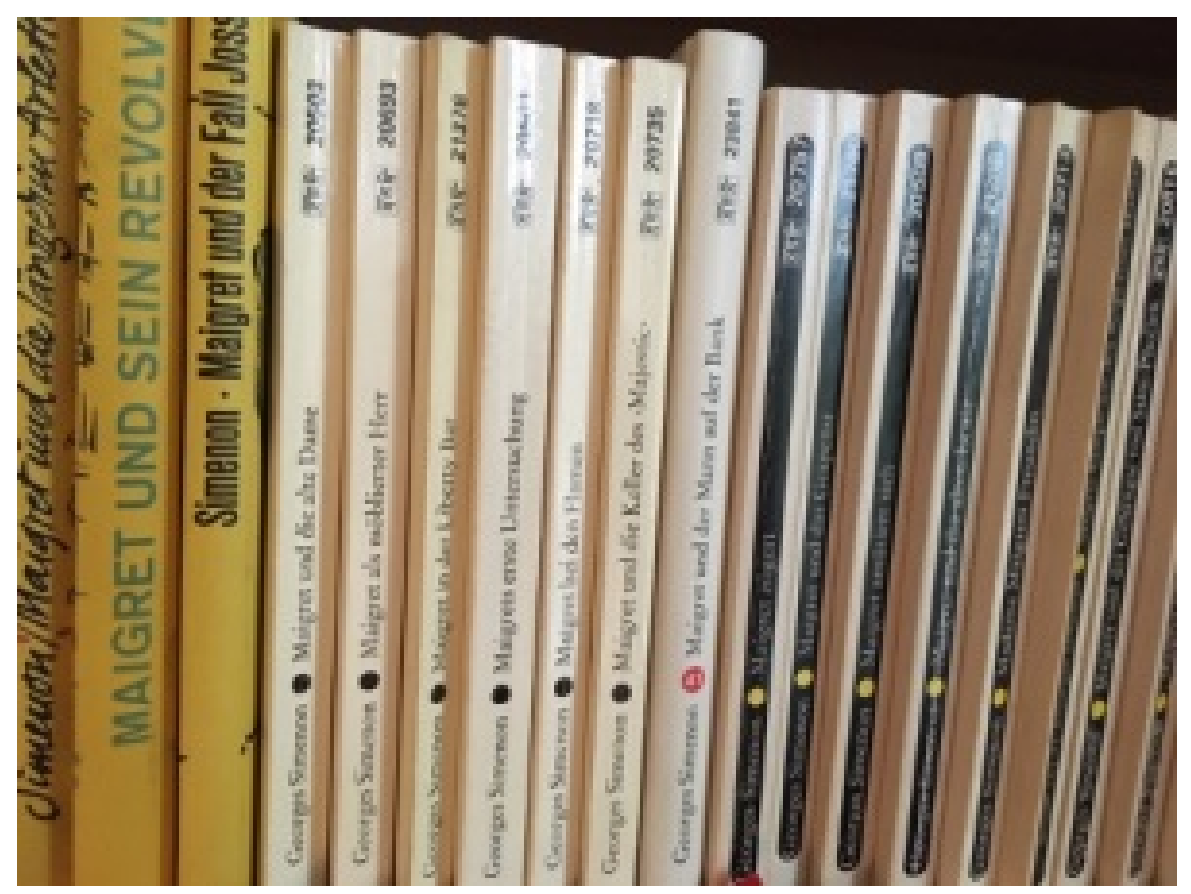

Foto: (C) Julika Griem

Mein unsystematisches Sammeln lässt mich auch Jules Maigret auf besondere Weise kennen lernen - in einer erratischen Rekonstruktion der Entwicklung dieses ikonischen Kommissars, die die Frage aufwirft, ob ich mir Notizen machen sollte, um einen Überblick zu behalten. Aber vielleicht kommt es darauf gar nicht an. Sondern eher auf ein regelmäßiges Wiedersehen unter minimal veränderten Bedingungen, um eine kleinteilige Synchronisation von Romanwelt und Leser*innenwelt, die wiederum Einsichten in die alltägliche Integration von Lektüreprozessen ermöglichen könnte. Auch hier spielt die allmähliche Anreicherung des Bekannten in Form einer neuen Routine eine wichtige Rolle. Weil ich mich in der pandemischen Krisensituation von professionellen Prägungen zu befreien können glaube, überlasse ich mich einem luxurierenden Verharren bei Details, die nicht unbedingt exegetisch nobilitierbar sind; freue mich an rituell platzierten Details wie den Bieren und belegten Broten aus der Brasserie Dauphine in Nachtschichten, dem anachronistischen Kachelofen in Maigrets Büro, den Beschreibungen seiner häufig eingenommenen Mahlzeiten, an Ticks und Marotten, den regelmäßigen Besuchen beim Ehepaar Pardon, den weit geöffneten Fenstern in der von Madame Maigret penibel gepflegten Wohnung am Boulevard Richard-Lenoir. Mit dieser repetitiv verstetigten Ausstattung erschafft Simenon eine durch Lesen „bewohnbare“ Welt, die mich vor dem Hintergrund des Dauerrauschens der Corona-Kommunikation immer noch nicht langweilt.

Und zu denken gibt. Steht Maigret auf der Seite der kleinen Leute, für die er immer wieder Mitgefühl zeigt und Grenzen überschreitet? Ist dies nicht einfach nur seinem Paternalismus geschuldet, der ihn auch als Ehemann einer weitgehend schweigend fürsorgenden Ehefrau charakterisiert? Verharren Simenons gerühmte MilieuBeschreibungen in einer kleinbürgerlichen Hölle, mit der zeittypische Manifestationen von Misogynie und Antisemitismus in Kauf zu nehmen sind? Diese Leseeindrücke beleuchten ein Spannungsverhältnis, das den Umgang mit kanonisiertem Material bestimmt: Auch dieser Krimi-Klassiker reorganisiert Nähe 
und Ferne, Bekanntes und Fremdes; lässt mich eine beschriebene Welt als vertraut empfinden, deren ideologische und habituelle Prämissen mir für meinen Alltag suspekt sind. So fragen Klassiker und Kanones ja gerade danach, wie Gegenwart und Vergangenheit im vergegenwärtigenden Akt des Lesens überhaupt aufeinander zu beziehen sind. Sicherlich nicht einfach nur im Sinne einer auf Identifikation und Empathie abgerichteten Aktualisierung. Die von Tag zu Tag beobachtete Immersion in die erzählte Welt Maigrets katapultiert die Leserin nicht einfach ins Paris der frühen Dekaden des 20. Jahrhunderts, und sie macht den massigen Ermittler schon gar nicht zum Lotsen, der beim Durchhalten im Lockdown hilft. Meine Lektüre akzentuiert vielmehr eine Oszillation zwischen dem gegenwärtigen Notstand und der topischen Krise, die die Kriminalliteratur als gut geölte generische Maschine immer wieder konstatiert, um den Anlass zur vorläufigen Wiederherstellung von Ordnung reproduzieren und variieren zu können.

Hin und her schwankt auch Kommissar Maigret selbst - der als ein Felsblock von Mann beschrieben wird, aber nicht darauf verzichtet, durch seine Tatorte und Milieus zu schweifen. In seiner sehr lesenswerten Studie „Rätsel und Komplotte“ hat der französische Soziologe Luc Boltanski die anglo-amerikanische und die französische Tradition gegenübergestellt: Dort die Trennung von Angestellten und freiem Amateur-Ermittler, hier der Polizeibeamte in staatlichen Diensten. Allerdings ist Maigret, so Boltanski, durchaus in sich gespalten. Er testet nicht nur im Umgang mit den großbürgerlich sozialisierten Richtern seine Freiräume aus, sondern fühlt sich auch aus biographischen Gründen einer anderen Sozialfigur nahe: Nach dem Tod seines Vaters musste Maigret sein Medizinstudium abbrechen und fing bei der Polizei an. Die regelmäßigen Gespräche mit seinem engsten Freund, dem Arzt Dr. Pardon, geben ihm die Gelegenheit, über eine Wahlverwandtschaft zwischen Medizin und Polizeiarbeit zu sinnieren. Boltanski leitet aus dieser Selbstwahrnehmung des Kommissars noch eine weitere Analogie ab, die sich in der gegenwärtigen Lage zu überdenken lohnt:

„Müssen nicht auch die Polizisten - wie die Mediziner, die gegen Mitte des 19. Jahrhunderts eine ganz zentrale biopolitische Rolle einnehmen, weil sie mit den Notaren und den Priestern als einzige den Abstand zwischen dem sexuellen, biologischen und psychologischen Intimbereich der individuellen und familiären Existenzen und der im Staat verkörperten Allgemeinheit überwinden können - die Menschen, das heißt die Verdächtigen, so genau wie möglich durchleuchten, um die Verteidigung des öffentlichen Wohls zu gewährleisten?“

Ob diese Engführung von medizinischer und polizeilicher Ermittlungsarbeit den besten Grund liefert, um in der gegenwärtigen pandemischen Ausnahmesituation 75 Maigret-Romane zu lesen, bleibt zu überdenken.Für eine Überprüfung gegenwärtiger Lese-Routinen spricht aber noch ein etymologisch-systematischer Zusammenhang: Das paradoxal aufgeladene Wort „Krise“ wurde im 16. Jahrhundert vom lateinischen „crisis“ ins Deutsche übernommen und in medizinischen Kontexten für den ,kritischen' Umschlagpunkt reserviert, an dem die Abwehr eines Infektionsprozesses 
möglich erscheint. Zur älteren Wortgeschichte gehören das griechische „krisis“ und „krinein“, im Substantiv eine „Beurteilung“ oder „Entscheidung“, die mit dem Verb für „trennen“ und „unterscheiden“, auch im Sinne einer „Kritik“, verbunden sind. Zur Geschichte von Krisen und unseren Gewohnheiten ihrer Deutung gehören somit ganz unterschiedliche Datensammler und Spurenleser zwischen Verwaltung und Medizin, Krankenhäusern und Kommissariaten, Lebens-, Gesellschafts- und Textwissenschaft. Walter Benjamin gilt als leidenschaftlicher Leser der Maigret-Romane. Er hat diese allerdings nur in seinen Briefen kommentiert, so dass es auch hier noch LeseGewohnheiten zu entdecken gibt, die zu einer genaueren Unterscheidung von Regeln und Ausnahmen führen könnten.

\section{References}

1. Auf eine ähnliche Idee kam offenbar der britische Romancier John Lanchester, der sich vor kurzem im London Review of Booksebenfalls intensiv mit den Maigret-Romanen befasst hat: https://www.lrb.co.uk/the-paper/v42/n11/johnlanchester/maigret-s-room.

2. So der Titel des vielgelesenen Blogs des Schriftstellers Wolfang Herrndorf.

3. Vgl. das Interview, das Carvel Collins 1955 für Paris Match mit Georges Simenon geführt hat: https://www.theparisreview.org/interviews/5020/theart-of-fiction-no-9-georges-simenon.

4. Vgl. auch Robert C. Courtine. Simenon und Maigret bitten zu Tisch: Die klassischen französischen Bistrorezepte der Madame Maigret. Zürich: Diogenes, 2009.

5. Vgl. Moritz Baßler, „Bewohnbare Strukturen und der Bedeutungsverlust des Narrativs. Überlegungen zur Serialität am Gegenwarts-Tatort." In: Christian Hißnauer, Stefan Scherer, Claudia Stockinger (Hgg.). Zwischen Serie und Werk. Fernseh- und Gesellschaftsgeschichte im »Tatort«. Bielefeld: transcript, 2015: 151-168.

6. Dass diese Aspekte der Maigret-Romane aus heutiger Sicht Probleme bereiten können, zeigt sich an den Verfilmungen des Senders ITV mit Rowan Atkinson aus den Jahren 2016 und 2017, in denen viele zeittypische Beschreibungsweisen und Figurendarstellungen abgemildert bzw. verändert wurden. Besonders deutlich treten antisemitische Züge z.B. in Pietr der Lette und Maigret und der Verrückte von Bergerac hervor; und Simenons Nähe zur französischen Kollaboration ist dokumentiert. Ein Großteil der Frauenfiguren wird in sexistischer Reduktion auf hervorgehobene physische Charakteristika eingeführt.

7. So z.B. in Maigret Maigret stellt eine Falle.

8. Luc Boltanski. Rätsel und Komplotte. Kriminalliteratur, Paranoia, moderne Gesellschaft. Frankfurt: Suhrkamp, 2015: 182-183. 
SUGGESTED CITATION: Griem, Julika: Auf ein Bier mit Kommissar Maigret, in: KWI-BLOG, [https://blog.kulturwissenschaften.de/auf-ein-bier-mit-kommissarmaigret/l, 29.06.2020

DOI: https://doi.org/10.37189/kwi-blog/20200629-0900 
Dieser Text wird über DuEPublico, dem Dokumenten- und Publikationsserver der Universität Duisburg-Essen, zur Verfügung gestellt. Die hier veröffentlichte Version der EPublikation kann von einer eventuell ebenfalls veröffentlichten Verlagsversion abweichen.

DOI:

10.37189/kwi-blog/20200629-0900

URN: urn:nbn:de:hbz:464-20200629-091050-4 